\title{
A REVIEW OF 493 RADIOGRAPHS OF DOMESTIC DOGS AND CATS FOR NEOPLASIA AND NEOPLASIA SUSPICIOUS SIGNS
}

doi: $10.2478 /$ rojost-2018-0044

D. Lescai

"Spiru Haret" University of Veterinary Medicine, Bucharest, Romania

Cats and dogs are routinely diagnosed with different forms of neoplasia. One of the simplest imaging techniques is radiography. This way one can assess neoplastic extent affecting soft and bone tissue. 493 radiographs from 235 dogs and cats have been reviewed.

372 radiographs for 94 dogs, an average of 5.2 images for every dog and 121 radiographs for 48 cats, an average of 2.5 images for each cat were done. 27 relevant or suspect radiographs were done in dogs, for a total of 14 neoplastic subjects, corresponding to 6 relevant or suspect images for a total of 3 neoplastic processes in cats. This is translated into a proportion of $7.25 \%$ relevant or suspect radiographs in dogs and $4.95 \%$ in cats. It also means that $14.89 \%$ of the dogs that require radiographic examination and $2.47 \%$ of the cats are suffering or are suspicious for neoplastic disease. 5 out of $14(35 \%)$ dogs had relevant radiographs for bone tissue neoplasia, 1 dog (7\%) for muscular neoplasia and 4 dogs (28.5\%) were suspicious for digital (mixed soft tissue/ bone tissue) neoplasia. 1 out of 3 cats had relevant images for muscular neoplasia and 2 cats for maxillary/ acetabular neoplasia.

Keywords: radiography, dogs, cats, musculoskeletal, neoplasia 